Review Article

\title{
Biological Effects of Medicinal Plants on Induced Periodontitis: A Systematic Review
}

\author{
Jefferson Soares de Oliveira, ${ }^{1}$ Moara e Silva Conceição Pinto, ${ }^{2}$ \\ Lucas de Araújo de Bastos Santana, ${ }^{1}$ Antonione Santos Bezerra Pinto, ${ }^{3}$ \\ David di Lenardo, ${ }^{1}$ and Daniel Fernando Pereira Vasconcelos ${ }^{2}$ \\ ${ }^{1}$ Laboratory of Biology and Biochemistry Plants (BIOqPLANT), Federal University of Piaui, Parnaiba, PI, Brazil \\ ${ }^{2}$ Laboratory of Histological Analysis and Prepare (LAPHIS), Federal University of Piaui, Parnaiba, PI, Brazil \\ ${ }^{3}$ Department of Morphology, LABICONTE, Federal University of Ceara, Fortaleza, CE, Brazil
}

Correspondence should be addressed to Daniel Fernando Pereira Vasconcelos; vasconcelos@ufpi.edu.br

Received 1 June 2016; Revised 22 June 2016; Accepted 23 June 2016

Academic Editor: Gul Atilla

Copyright (C) 2016 Jefferson Soares de Oliveira et al. This is an open access article distributed under the Creative Commons Attribution License, which permits unrestricted use, distribution, and reproduction in any medium, provided the original work is properly cited.

\begin{abstract}
Objective. The aim of this systematic review was to investigate the advances in the study of medicinal plants and their biologic effects on periodontitis in animal models. Study Design. A systematic search was conducted by three independent researchers, who screened articles published up to March/2016, to identify the studies that contained sufficient and clear information on the association of the medicinal plants and periodontitis in murine models. The searches were performed using PubMed, Cochrane, and Science Direct databases. Results. After a critical analysis of titles and abstracts, 30 studies were finally eligible for analysis. The studies presented a great diversity of the experiment designed regarding the methods of induced periodontitis and the evaluation of the medicinal plants efficacy. None of the studies described the possible toxic effects associated with the administration of the plant material to animals and whether they could prevent damage to organs caused by systemic effect of induced periodontitis. Gel-based formulations containing plant substances are seen as an interesting strategy to treat periodontitis. Conclusions. In this systematic review, the state-of-the-art knowledge on the medicinal plants and the induced periodontitis was critically evaluated and discussed from the experiment designed to the possible clinical application.
\end{abstract}

\section{Introduction}

Periodontitis is one of the most extensive oral problems that affect human population, resulting from an inflammatory response against microorganisms involved with plaque accumulation on the subgingival dental surface. The development of the process depends on the interaction between the bacteria in the site of infection. The presence of an oral biofilm composed by bacteria and their products includes also lipopolysaccharides and proteinases that are responsible for the progression of periodontitis. Bacteria stimulate host immunopathological and inflammatory mechanisms that result in the destruction of the periodontal tissue [1].

Different strategies have been used to treat periodontal diseases. Mechanical therapy and surgical procedures reduce microbial burden, being effective in the control of the periodontitis progression. Nevertheless, this regulation is not always satisfactory, possibly due to the prominent role of immunogenetic response on periodontal destruction. In some cases, adjunctive therapies may be required [2]. Thus, the discovery and development of potential therapeutic drugs with the ability to regulate the host immune and bacteria-mediated inflammatory interactions are a valuable approach for the prevention and treatment of the periodontal disease [2-4]. In this context, plants can represent an interesting source of molecules with a potential activity against periodontal disease progression. The growing incidence of periodontitis, the increased resistance of oral bacteria to antibiotics, and the adverse effects of some drugs used in dentistry all motivate the search for safe and effective molecules to treat and prevent the disease [5]. 
Medicinal plants have been fundamental for thousands of years to provide bioactive molecules used to treat different types of human infirmities, such as inflammation, pain, and tumors. Also, they can be a source of compounds to be tested in the treatment of periodontal diseases. Nowadays, there is an increasing number of scientific investigation exploring plant extracts or purified molecules in periodontal diseases [3]. Several of these studies were performed using animal models since biochemical, histological, and anatomical features are similar to humans $[3,4,6,7]$.

The aim of this systematic review was to investigate the advances in the study of medicinal plants and the development of induced periodontitis in animal models. Data were described and discussed in order to evaluate the limitation and also the perspectives of the application of these agents in the treatment of the periodontal disease.

\section{Material and Methods}

A systematic search was conducted by three independent researchers who screened articles published up to March/2016 in order to identify the studies that contained sufficient and clear information on the association of the medicinal plants and periodontitis in murine models. The searches were performed using PubMed (http://www.ncbi .nlm.nih.gov/pubmed), Cochrane (http://www.cochranelibrary.com/), and Science Direct (http://www.sciencedirect .com/) databases using the key words plant, periodontitis, and rats and search details "plant" [All Fields] AND "periodontitis" [All Fields] AND "rats" [All Fields]. The articles selected by each researcher were compared to remove duplicate records and 109 different articles were initially evaluated. After a critical analysis of titles and abstracts, 79 articles were excluded such as articles that were not in English, articles that were not fully available, or those that did not report an association between medicinal products and murine model of periodontitis. Hence, 30 studies were finally eligible for a qualitative analysis for this review (Figure 1).

\section{Results and Discussion}

Table 1 summarize plant species, plant material, route of administration, animal used, type of induction and time of analysis, and the ability of the medicinal plant to reduce alveolar bone loss related to 30 selected articles.

The majority of articles (43.3\%) described studies conducted with plant extracts, $40 \%$ with purified compounds and $16.6 \%$ with a mixture of two or more plant extracts. Among them, six articles described experiments developed using a commercial product [8-13], containing purified compounds or a mixture of plant extracts. None of authors mentioned the process of obtaining selected plant material as an important limitation for development of the research.

In general, studies were preferentially conducted using Rattus norvegicus (90\%) of different strains Wistar, Lewis, or Sprague-Dawley. This specie is one of the mostly used for in vivo experimental models, including the pathogenesis of periodontal disease because they offer some advantages such as price, easy handling, and the possibility of microbiological,

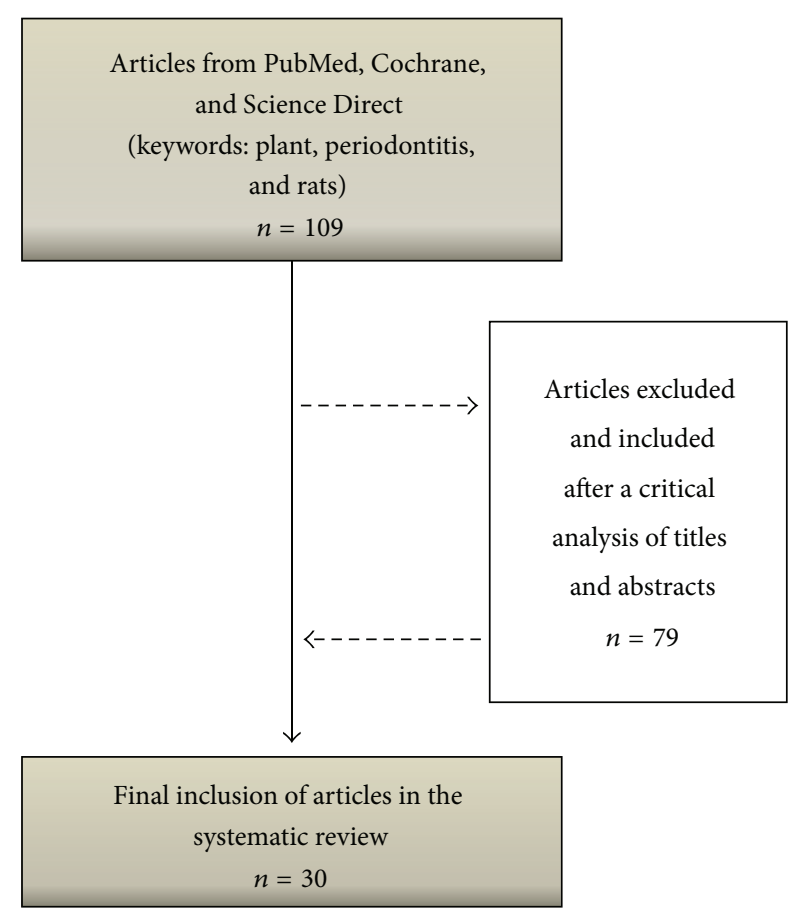

FIGURE 1: Search flowchart and selection of articles for the review of the literature.

macroscopic, and histological evaluation [14]. Other studies were conducted using Mus musculus Balb/c (6.6\%) or C57BL/6. Rodents were preferred as the animal model since they present biochemical, histological, and anatomical features similar to humans [14].

Taking into consideration the sex of the animal, most authors chose to use males (86.6\%). Two articles described the use of females $[12,15]$ and two studies were conducted using males and females $[4,8]$. One study did not describe the sex of the animals [16]. Most in vivo studies which investigate the biological effect of plant substances favor the use of male animals. This choice is based on the fact that female hormones should interfere in the development and progression of the disease [17]. None of the articles analyzed was related to the hormonal influence on the biological activities investigated. However, studies conducted with females could clarify the effect of their hormones on the specific pathways involved in the periodontitis progression.

Regarding the common type of periodontitis-induction, in $66.6 \%$ of the articles, the process was caused by ligature (silk: $23.3 \%$, nylon: $23.3 \%$, and cotton: $20 \%$ ). This method is widely used because it facilitates the accumulation of biofilm. This procedure increases the infiltration of inflammatory cells and the production of chemical mediators that lead to the degradation of the tissues around the teeth contributing to the destruction of the periodontal tissues [1]. In addition to periodontitis induced by ligature, $16.6 \%$ of articles described the gingival injection of bacteria, such as $P$. gingivalis and $16.6 \%$ described the administration of E. coli endotoxin (LPS) or S. griseus (proteases) to promote the periodontal disease. The inoculation of bacteria or their subproducts leads to 


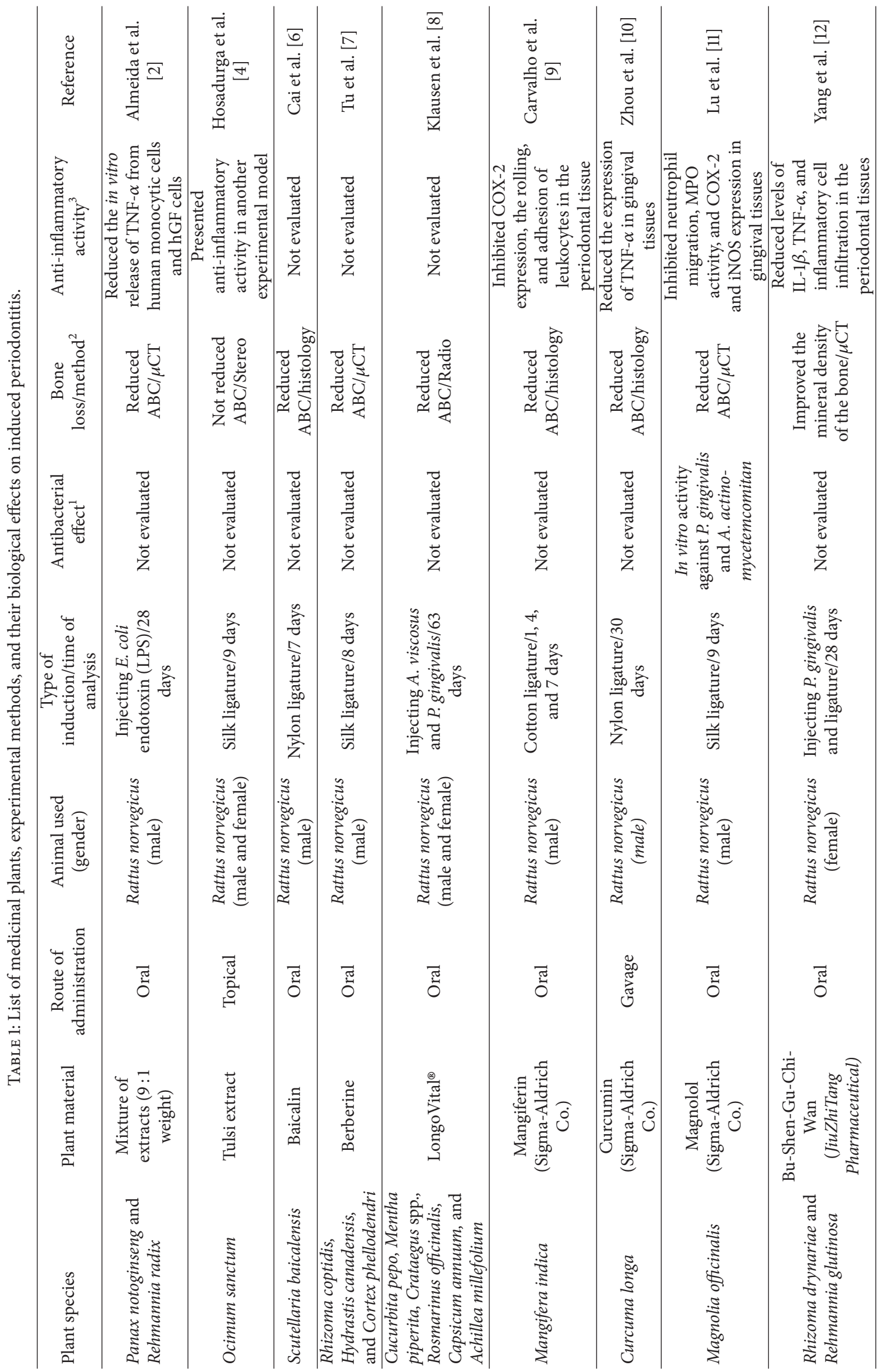




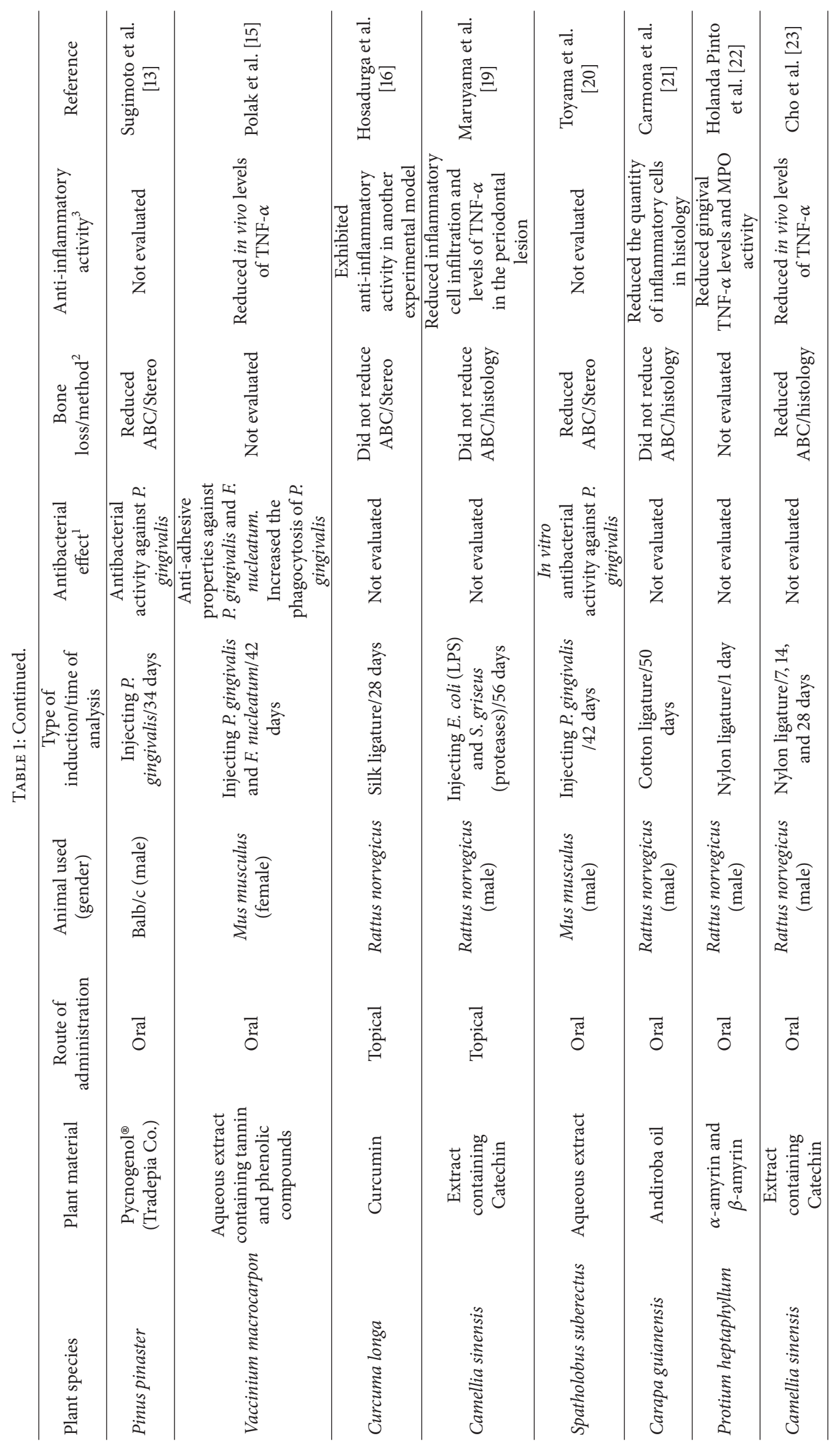




\begin{tabular}{|c|c|c|c|c|c|c|c|c|c|}
\hline 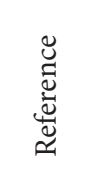 & 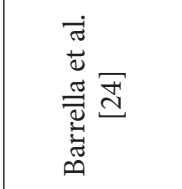 & 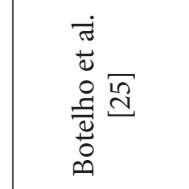 & 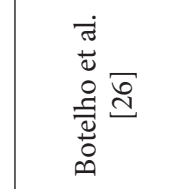 & 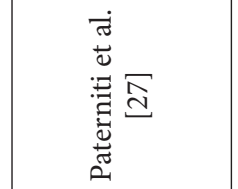 & 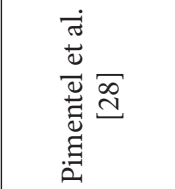 & 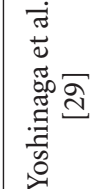 & 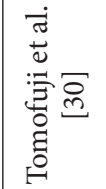 & 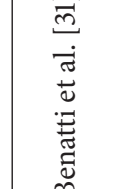 & 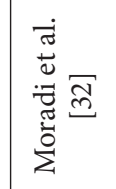 \\
\hline 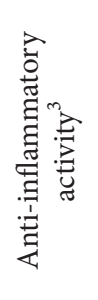 & 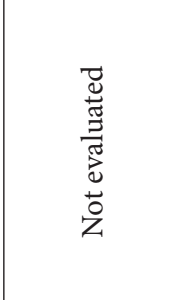 & 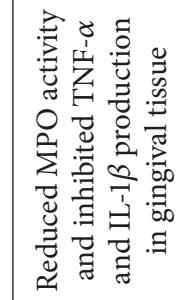 & 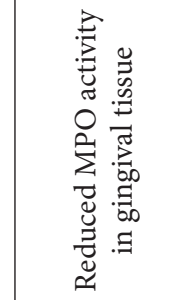 & 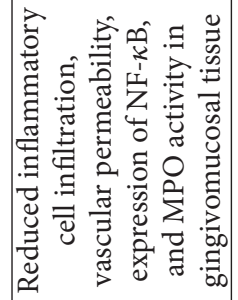 & 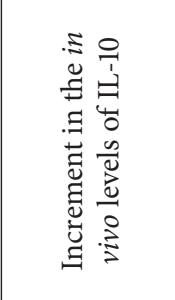 & 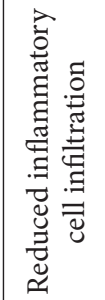 & 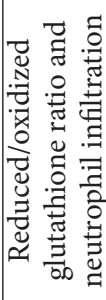 & 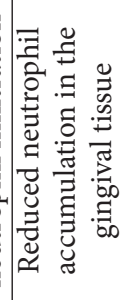 & 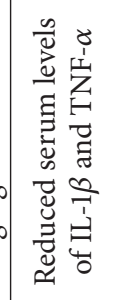 \\
\hline 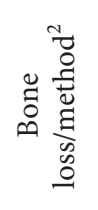 & 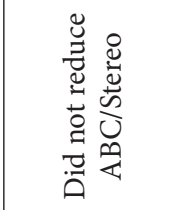 & 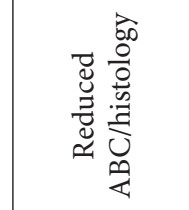 & 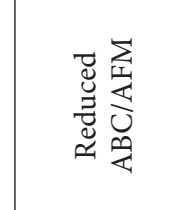 & 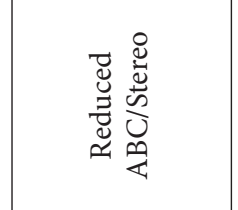 & 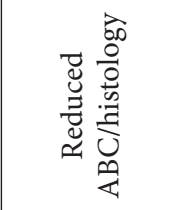 & 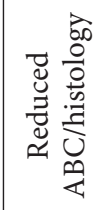 & 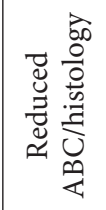 & 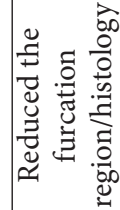 & 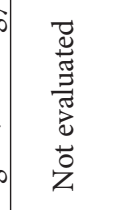 \\
\hline 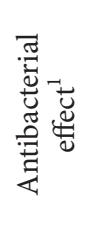 & 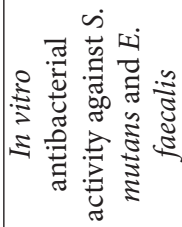 & 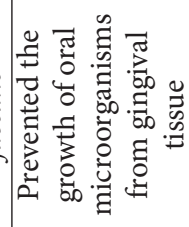 & 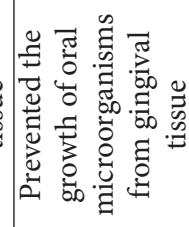 & 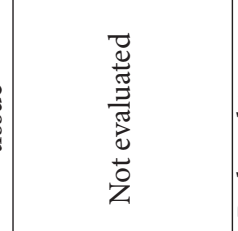 & 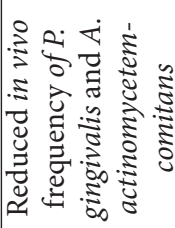 & 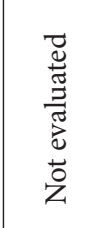 & 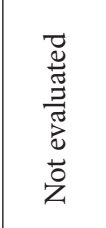 & 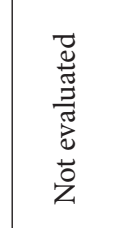 & 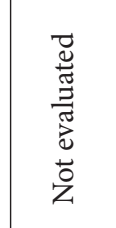 \\
\hline 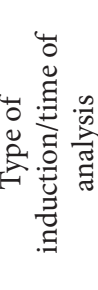 & 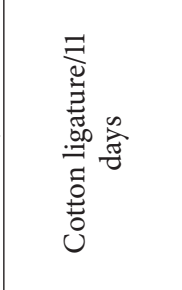 & 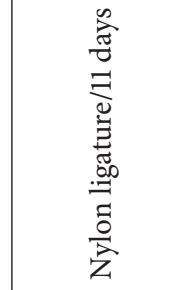 & 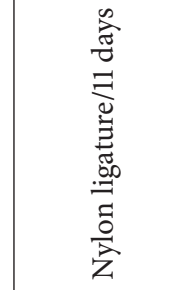 & 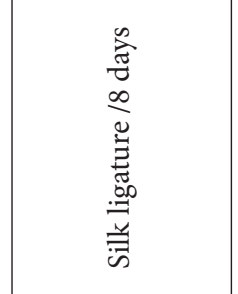 & 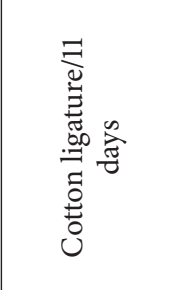 & 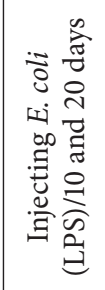 & 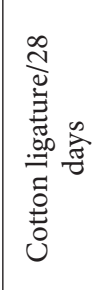 & 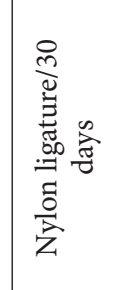 & 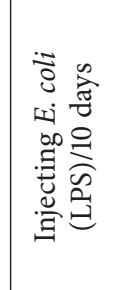 \\
\hline 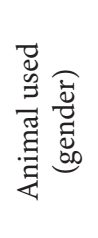 & 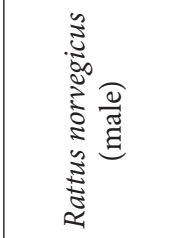 & 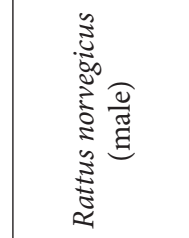 & 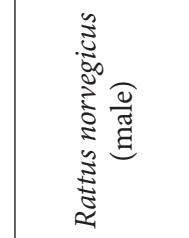 & 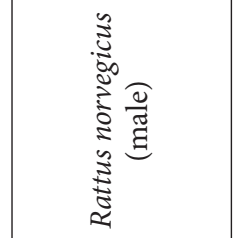 & 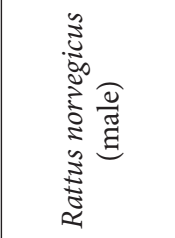 & 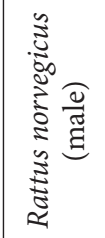 & 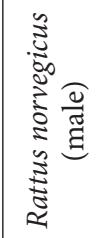 & 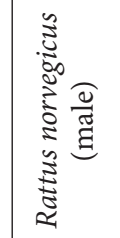 & 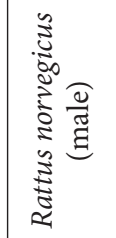 \\
\hline 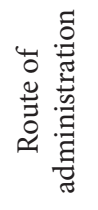 & 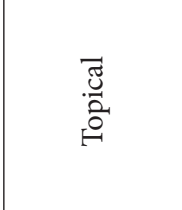 & 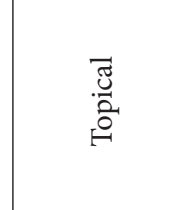 & 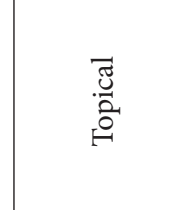 & $\overline{\widetilde{\sigma}}$ & 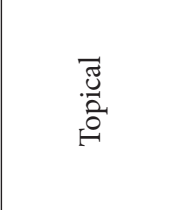 & $\begin{array}{l}\overline{\tilde{n}} \\
\overline{0}\end{array}$ & $\overline{\widetilde{g}}$ & 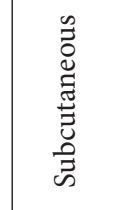 & 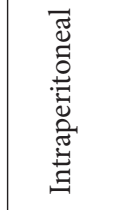 \\
\hline 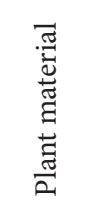 & 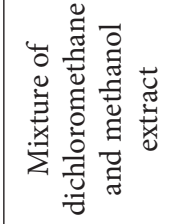 & 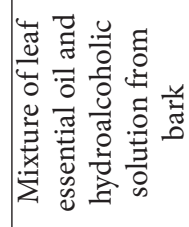 & 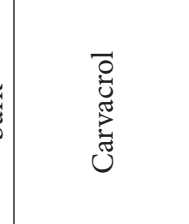 & 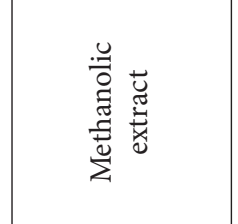 & 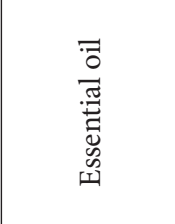 & 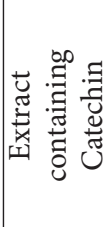 & 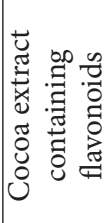 & 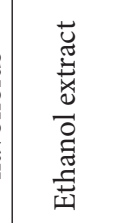 & $\stackrel{\Xi}{0}$ \\
\hline 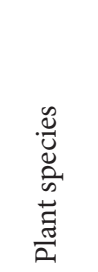 & 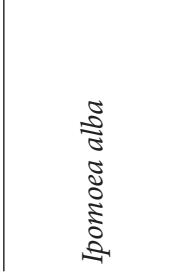 & 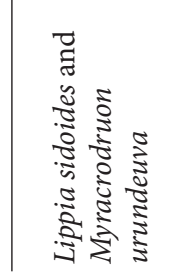 & 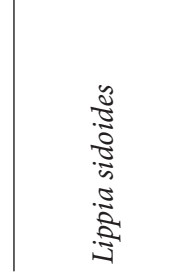 & 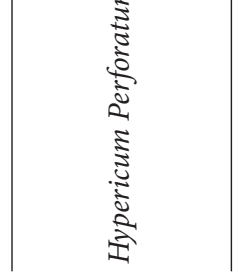 & 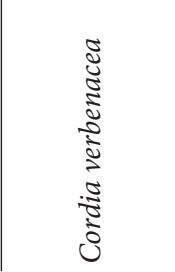 & 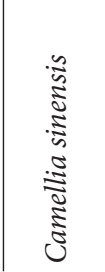 & 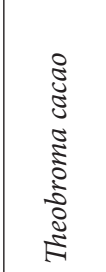 & 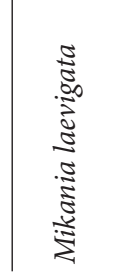 & 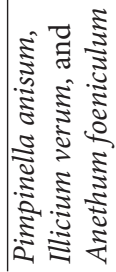 \\
\hline
\end{tabular}




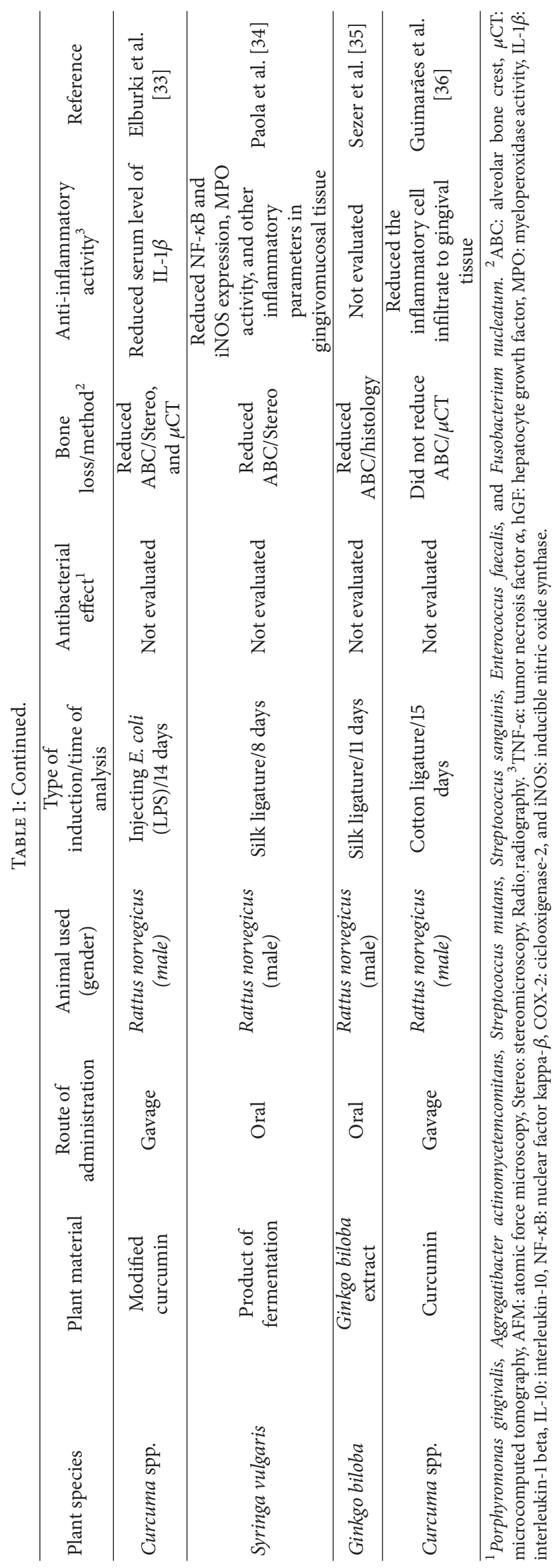


an inflammatory response different from that promoted by periodontitis induced through induction with ligature [18].

Some of the studies analyzed described that long periods of experimental design were utilized to properly investigate the severity of tissue destruction during periodontal disease treatment: 63 days [8]; 56 days [19]; 42 days [20]; and 50 days [21]. However, the time of analysis changed substantially. More than $55 \%$ of the studies performed experiments during one to two weeks, $26.6 \%$ for three to four weeks, and $13.3 \%$ during six weeks or more. One study was conducted with an acute periodontitis rat model in 24 hours [22]. These variations in the experimental periods may be due to factors such as the type and location of the ligature, the bacteria species or their sub-products injected for the induction of the disease and the specie (strain), and age and weight of the animal used. These factors are generally associated with the objective of the study and the expected results.

More than $66 \%$ of studies chose the oral administration to evaluate the efficacy of the plant material. The topical administration was described by $26.6 \%$ of the studies and $6.9 \%$ treated animals through subcutaneous or intraperitoneal cavity injections. The route of administration is an important parameter to influence the efficacy of the material since it can interfere with the sufficient amount of substance available to promote the biological effects. As observed, different results were seen for alveolar bone loss of animals submitted to the treatment with an extract containing Catechin obtained from Camellia sinensis. Although the route of administration had not influenced the anti-inflammatory activity of the material, a significant reduction of bone loss was observed when given orally [23] and no difference was seen after its topical treatment [19].

Periodontal disease initiation and progression occur as a consequence of the host response to microorganisms of the dental biofilm [1]. Therefore, the antibacterial effect is an important factor in the periodontal therapy. Only $26.6 \%$ of the studies investigated the capacity of the material to present antibacterial activity. In the study developed by Barrella et al. [24] the organic extract obtained from Ipomoea alba showed significant in vitro activity against Streptococcus mutans, $S$. sanguinis, and Enterococcus faecalis. The commercial product Magnolol obtained from Magnolia officinalis exhibited intense inhibition of Porphyromonas gingivalis and Aggregatibacter actinomycetemcomitans growth in a dependent dose [11] and the aqueous extract from Vaccinium macrocarpon containing tannin and phenolic compounds inhibited the adhesion of Fusobacterium nucleatum and Porphyromonas gingivalis [15].Study developed by Botelho et al. [25] and Botelho et al. [26] with Lippia sidoides observed the decrease of salivary bacterial levels and this event was followed by an increment in clinical scores of gingival bleeding. Except for the work published by Klausen et al. [8] which did not evaluate the alveolar bone loss, all the articles that described the identification of antibacterial activity also demonstrated that plant material treatment induced a significant reduction of alveolar bone loss.

The alveolar bone loss is one of the most important parameters evaluated in the induced periodontal disease. From 30 selected articles, 27 evaluated the ability of the plant material to promote a significant reduction of the bone loss. Carvacrol purified from Lippia sidoides, the extract containing Catechin obtained from Camellia sinensis, the essential oil of Cordia verbenacea, and the methanolic extract of Hypericum perforatum are examples of $77 \%$ of studies that observed a significant reduction of the bone loss [23, $26-28]$. On the other hand, in $23 \%$ of studies such as andiroba oil from Carapa guianensis, as well as the mixture of dichloromethane and methanol extracts from Ipomoea alba and Longo Vital, the authors did not observe a significant effect [8, 21, 24]. Moreover, it was interesting to note that different studies using the same plant material have reached different results regarding alveolar bone loss. According to Yoshinaga et al. [29] the topical treatment of animals with the extract containing Catechin (Camellia sinensis) promoted a significant reduction of bone loss in periodontitis induced by E. coli (LPS). On the other hand, the same material did not present a significant reduction in the alveolar bone loss against periodontitis induced by injection of E. coli (LPS) and S. griseus (proteases) [19]. Different efficacy was also observed in studies developed with curcumin (Curcuma longa). The topical administration of curcumin did not reduce the alveolar bone loss [16] while its oral administration reduced this parameter significantly [10]. The methodologies used to evaluate the alveolar bone loss were histology (44.0\%), stereomicroscopy (25.9\%), microcomputed tomography $(22.2 \%)$, atomic force microscopy (3.7\%), and radiography (3.7\%). Histology, stereomicroscopy, and microcomputed tomography are favorable methods once they yield precise information concerning the evaluation of periodontal tissue destruction.

It is noteworthy that the reduction of the inflammatory process may be associated with a reduced bone loss $[12,30$, 31]. Because of this, a reduction of inflammatory process is usually investigated in periodontal disease. Taking the selected articles into account, $76.6 \%$ of them evaluated inflammatory parameters associated with induced periodontitis. The common strategies used to observe the ability of the plant material to inhibit the inflammatory process in periodontal tissue were the evaluation of migration of inflammatory cells (30\%), measurement of proinflammatory cytokines (TNF- $\alpha$ : 30\% and IL1- $\beta$ : 13.3\%) $[2,10,12,15,19,22$, $23,25,32,33]$, respectively, and dosage of myeloperoxidase (MPO) activity (20\%) [11, 22, 25-27, 34]. Other molecular markers of inflammatory process, such as iNOS (13.0\%) $[11,34], \mathrm{NF}-\kappa \mathrm{B}(8.7 \%)[27,34], \mathrm{COX}-2(8.7 \%)[9,11]$, and IL-10 (4.3\%) [28], were also investigated. Although the presence of the anti-inflammatory effect reflects a possible efficacy against periodontitis, $13 \%$ of the authors who found anti-inflammatory properties did not observe a significant reduction of alveolar bone loss in the periodontitis assays. The anti-inflammatory activity was not analyzed in the $23.3 \%$ $[6-8,13,20,24,35]$ of the studies. The andiroba oil (Carapa guianensis), the extract from Camellia sinensis (containing Catechin), and Curcumin (Curcuma spp.) were able to reduce the number of inflammatory cells in the gingival tissue. However, they were not effective to protect from destructive periodontal process $[19,21,36]$. According to the authors, these negative results may be related to the time delay to 
reach levels that are high enough for the biological effects of administered substances in the experimental models adopted. Further researches are necessary to observe how much time is appropriate to manage the substances for the recovery of bone loss.

Although there is a close relation between the bone reabsorption and the inflammatory response, we suggest that the negative results of some of substances analyzed should be attributed to the fact that they do not act in the osteoclastogenesis process. This information is supported by the personal observation conducted using sulphated polysaccharides recovered from red marine algae Gracilaria caudata. Our data revealed that the treatment of experimental animals with sulphated polysaccharides improved clinical and inflammatory parameters. However, no significant effect was observed in the reduction of alveolar bone loss (unpublished data).

It is important to mention that, although many plant substances may have deleterious effects to different animal organs [37], none of the evaluated studies has investigated the possible toxicological effects associated with the administration of the medicinal plants. In addition, several studies have documented that induced periodontitis is followed by significant changes of morphological structures and biochemical functions of different organs [38, 39]. Yet again, none of studies investigated the ability of these medicinal plants to prevent changes in organs promoted by a systemic action of the induced periodontitis.

Finally, 8 of the 30 selected articles conducted their experiments with a gel-based formulation containing the plant material investigated. In these experiments, the authors administered the gel topically, generally three times a day. The main idea behind these investigations is to reveal the further potential of the combined gel preparation to combat periodontal disease in closer than clinical situations. This was the outlook investigated in the studies published by $[40,41]$. The authors evaluated the efficiency of a green tea Catechin gel as an adjunct on human periodontal therapy. In first work [40], the authors observed a significant reduction on pockets and inflammation during the 4 weeks of the clinical trial. In the second work [41], it was demonstrated that when used as an adjunct to periodontal treatment, green tea gel could provide benefit in reducing bleeding on probing and gingival inflammation at 1st and 3rd months of evaluation. What makes these studies even more relevant is the fact that the findings on complementary products for the treatment of human periodontitis still can be enhanced.

\section{Conclusion}

In conclusion, the selected studies presented a large diversity of experimental designs, concerning the type of induction, time of analysis, and methods used for the evaluation of alveolar bone loss, anti-inflammatory, and antibacterial activities. None of the studies evaluated the possible toxic effects associated with the administration of the material analyzed or their ability to prevent damages to organs caused by systemic effects of induced periodontitis. Gel-based formulations present an interesting strategy to treat periodontitis; however, further studies are necessary to clarify its usefulness in the clinical situation.

\section{Competing Interests}

The authors declare that they have no conflict of interests.

\section{Acknowledgments}

This research is supported by the Federal University of Piauí (UFPI-Edital PIBIC 2014/2015 and BIAMA 03/2014), CNPq (455104/2014-0). The authors thank teacher Abilio Borghi for the grammar review of the paper.

\section{References}

[1] A. Bascones-Martínez, M. Muñoz-Corcuera, S. Noronha, P. Mota, C. Bascones-Ilundain, and J. Campo-Trapero, "Host defence mechanisms against bacterial aggression in periodontal disease: basic mechanisms," Medicina Oral, Patologia Oral y Cirugia Bucal, vol. 14, no. 12, pp. e680-e685, 2009.

[2] J. De Almeida, E. Ervolino, L. H. Bonfietti et al., "Adjuvant therapy with sodium alendronate for the treatment of experimental periodontitis in rats," Journal of Periodontology, vol. 86, no. 10, pp. 1166-1175, 2015.

[3] R.-Y. Huang, S.-H. Lu, K.-W. Su et al., "Diacerein: a potential therapeutic drug for periodontal disease," Medical Hypotheses, vol. 79, no. 2, pp. 165-167, 2012.

[4] R. R. Hosadurga, S. N. Rao, R. Edavanputhalath et al., "Evaluation of the efficacy of $2 \%$ Ocimum sanctum gel in the treatment of experimentalperiodontitis," International Journal of Pharmaceutical Investigation, vol. 5, no. 1, pp. 35-42, 2015.

[5] C. M. Ardila, M. A. López, and I. C. Guzmán, "High resistance against clindamycin, metronidazole and amoxicillin in Porphyromonas gingivalis and Aggregatibacter actinomycetemcomitans isolates of periodontal disease," Medicina Oral, Patologia Oral y Cirugia Bucal, vol. 15, no. 6, pp. e947-e951, 2010.

[6] X. Cai, C. Li, G. Du, and Z. Cao, "Protective effects of baicalin on ligature-induced periodontitis in rats," Journal of Periodontal Research, vol. 43, no. 1, pp. 14-21, 2008.

[7] H.-P. Tu, M. M. J. Fu, P.-J. Kuo et al., "Berberine's effect on periodontal tissue degradation by matrix metalloproteinases: an in vitro and in vivo experiment," Phytomedicine, vol. 20, no. 13, pp. 1203-1210, 2013.

[8] B. Klausen, A. Apostolopoulos, K. Stoltze, and F. Nörgaard, "Effect of LongoVital treatment on development of periodontal disease in rats," Scandinavian Journal of Dental Research, vol. 101, no. 1, pp. 33-36, 1993.

[9] R. R. Carvalho, C. H. Pellizzon, L. Justulin Jr. et al., "Effect of mangiferin on the development of periodontal disease: involvement of lipoxin A4, anti-chemotaxic action in leukocyte rolling," Chemico-Biological Interactions, vol. 179, no. 2-3, pp. 344-350, 2009.

[10] T. Zhou, D. Chen, Q. Li, X. Sun, Y. Song, and C. Wang, "Curcumin inhibits inflammatory response and bone loss during experimental periodontitis in rats," Acta Odontologica Scandinavica, vol. 71, no. 2, pp. 349-356, 2013.

[11] S.-H. Lu, R.-Y. Huang, and T.-C. Chou, "Magnolol ameliorates ligature-induced periodontitis in rats and osteoclastogenesis: in vivo and in vitro study," Evidence-Based Complementary and 
Alternative Medicine, vol. 2013, Article ID 634095, 12 pages, 2013.

[12] H. Yang, Q. Wen, J. Xue, and Y. Ding, "Alveolar bone regeneration potential of a traditional Chinese medicine, Bu-Shen-GuChi-Wan, in experimental periodontitis," Journal of Periodontal Research, vol. 49, no. 3, pp. 382-389, 2014.

[13] H. Sugimoto, K. Watanabe, T. Toyama et al., "Inhibitory effects of French pine bark extract, pycnogenol ${ }^{\circledR}$, on alveolar bone resorption and on the osteoclast differentiation," Phytotherapy Research, vol. 29, no. 2, pp. 251-259, 2015.

[14] X. Struillou, H. Boutigny, A. Soueidan, and P. Layrolle, "Experimental animal models in periodontology: a review," The Open Dentistry Journal, vol. 4, no. 1, pp. 33-47, 2010.

[15] D. Polak, R. Naddaf, L. Shapira, E. I. Weiss, and Y. HouriHaddad, "Protective potential of non-dialyzable material fraction of cranberry juice on the virulence of $P$. gingivalis and $F$. nucleatum mixed infection," Journal of Periodontology, vol. 84, no. 7, pp. 1019-1025, 2013.

[16] R. R. Hosadurga, S. Rao, J. Jose, N. C. Rompicharla, M. Shakil, and R. Shashidhara, "Evaluation of the efficacy of $2 \%$ curcumin gel in the treatment of experimental periodontitis," Pharmacognosy Research, vol. 6, no. 4, pp. 326-333, 2014.

[17] E. Figuero, A. Carrillo-De-Albornoz, D. Herrera, and A. Bascones-Martínez, "Gingival changes during pregnancy: I. Influence of hormonal variations on clinical and immunological parameters," Journal of Clinical Periodontology, vol. 37, no. 3, pp. 220-229, 2010.

[18] S. Garcia de Aquino, F. R. Manzolli Leite, D. R. Stach-Machado, J. A. Francisco da Silva, L. C. Spolidorio, and C. Rossa Jr., "Signaling pathways associated with the expression of inflammatory mediators activated during the course of two models of experimental periodontitis," Life Sciences, vol. 84, no. 21-22, pp. 745-754, 2009.

[19] T. Maruyama, T. Tomofuji, Y. Endo et al., "Supplementation of green tea catechins in dentifrices suppresses gingival oxidative stress and periodontal inflammation," Archives of Oral Biology, vol. 56, no. 1, pp. 48-53, 2011.

[20] T. Toyama, K. Todoki, Y. Takahashi et al., "Inhibitory effects of Jixueteng on P. gingivalis-induced bone loss and osteoclast differentiation," Archives of Oral Biology, vol. 57, no. 11, pp. 15291536, 2012.

[21] G. B. Carmona, R. K. C. Teixeira, M. V. H. Brito et al., "Effect of andiroba oil on periodontitis in wistar rats," Acta Cirurgica Brasileira, vol. 28, no. 6, pp. 430-434, 2013.

[22] S. A. Holanda Pinto, L. M. S. Pinto, G. M. A. Cunha, M. H. Chaves, F. A. Santos, and V. S. Rao, "Anti-inflammatory effect of $\alpha, \beta$-Amyrin, a pentacyclic triterpene from Protium heptaphyllum in rat model of acute periodontitis," Inflammopharmacology, vol. 16, no. 1, pp. 48-52, 2008.

[23] A.-R. Cho, J.-H. Kim, D.-E. Lee et al., "The effect of orally administered epigallocatechin-3-gallate on ligature-induced periodontitis in rats," Journal of Periodontal Research, vol. 48, no. 6, pp. 781-789, 2013.

[24] G. E. Barrella, I. B. Suffredini, F. V. Ribeiro, F. R. Cirano, and S. P. Pimentel, "Evaluation of the effect of an organic extract obtained from Ipomoea alba L. on experimental periodontitis in rats," Brazilian Oral Research, vol. 26, no. 2, pp. 158-164, 2012.

[25] M. A. Botelho, V. S. Rao, C. B. M. Carvalho et al., "Lippia sidoides and Myracrodruon urundeuva gel prevents alveolar bone resorption in experimental periodontitis in rats," Journal of Ethnopharmacology, vol. 113, no. 3, pp. 471-478, 2007.
[26] M. A. Botelho, J. G. Martins, R. S. Ruela et al., "Protective effect of locally applied carvacrol gel on ligature-induced periodontitis in rats: a tapping mode AFM study," Phytotherapy Research, vol. 23, no. 10, pp. 1439-1448, 2009.

[27] I. Paterniti, E. Briguglio, E. Mazzon et al., "Effects of Hypericum Perforatum, in a rodent model of periodontitis," BMC Complementary and Alternative Medicine, vol. 10, article 73, 2010.

[28] S. P. Pimentel, G. E. Barrella, R. C. V. Casarin et al., "Protective effect of topical Cordia verbenacea in a rat periodontitis model: immune-inflammatory, antibacterial and morphometric assays," BMC Complementary and Alternative Medicine, vol. 12, no. 1, article 224, pp. 1-8, 2012.

[29] Y. Yoshinaga, T. Ukai, S. Nakatsu et al., "Green tea extract inhibits the onset of periodontal destruction in rat experimental periodontitis," Journal of Periodontal Research, vol. 49, no. 5, pp. 652-659, 2014.

[30] T. Tomofuji, D. Ekuni, K. Irie et al., "Preventive effects of a cocoa-enriched diet on gingival oxidative stress in experimental periodontitis," Journal of Periodontology, vol. 80, no. 11, pp. 1799-1808, 2009.

[31] B. B. Benatti, J. C. Campos-Júnior, V. J. Silva-Filho et al., "Effects of a Mikania laevigata extract on bone resorption and RANKL expression during experimental periodontitis in rats," Journal of Applied Oral Science, vol. 20, no. 3, pp. 340-346, 2012.

[32] J. Moradi, F. Abbasipour, J. Zaringhalam et al., "Anethole, a medicinal plant compound, decreases the production of proinflammatory TNF- $\alpha$ and IL- $1 \beta$ in a rat model of LPS-induced periodontitis," Iranian Journal of Pharmaceutical Research, vol. 13, no. 4, pp. 1319-1325, 2014.

[33] M. S. Elburki, C. Rossa, M. R. Guimaraes et al., "A novel chemically modified curcumin reduces severity of experimental periodontal disease in rats: Initial observations," Mediators of Inflammation, vol. 2014, Article ID 959471, 10 pages, 2014.

[34] R. D. I. Paola, G. Oteri, E. Mazzon et al., "Effects of verbascoside, biotechnologically purified by Syringa vulgaris plant cell cultures, in a rodent model of periodontitis," Journal of Pharmacy and Pharmacology, vol. 63, no. 5, pp. 707-717, 2011.

[35] U. Sezer, M. İ. Kara, K. Erciyas et al., "Protective effects of ginkgo biloba extract on ligature-induced periodontitis in rats," Acta Odontologica Scandinavica, vol. 71, no. 1, pp. 38-44, 2013.

[36] M. R. Guimarães, L. S. Coimbra, S. G. de Aquino, L. C. Spolidorio, K. L. Kirkwood, and C. Rossa Jr., "Potent antiinflammatory effects of systemically administered curcumin modulate periodontal disease in vivo," Journal of Periodontal Research, vol. 46, no. 2, pp. 269-279, 2011.

[37] U. F. Ezuruike and J. M. Prieto, "The use of plants in the traditional management of diabetes in Nigeria: pharmacological and toxicological considerations," Journal of Ethnopharmacology, vol. 155, no. 2, pp. 857-924, 2014.

[38] T. Tomofuji, D. Ekuni, R. Yamanaka et al., "Chronic administration of lipopolysaccharide and proteases induces periodontal inflammation and hepatic steatosis in rats," Journal of Periodontology, vol. 78, no. 10, pp. 1999-2006, 2007.

[39] T. Tomofuji, T. Sanbe, D. Ekuni et al., "Oxidative damage of rat liver induced by ligature-induced periodontitis and chronic ethanol consumption," Archives of Oral Biology, vol. 53, no. 12, pp. 1113-1118, 2008.

[40] V. K. Chava and B. D. Vedula, "Thermo-reversible green tea catechin gel for local application in chronic periodontitis: a 4- 
week clinical trial," Journal of Periodontology, vol. 84, no. 9, pp. 1290-1296, 2013.

[41] K. Rattanasuwan, S. Rassameemasmaung, V. Sangalungkarn, and C. Komoltri, "Clinical effect of locally delivered gel containing green tea extract as an adjunct to non-surgical periodontal treatment," Odontology, vol. 104, no. 1, pp. 89-97, 2016. 


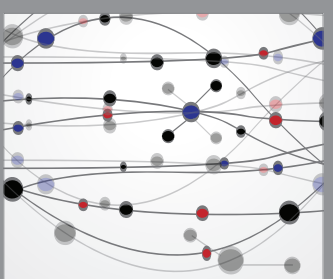

The Scientific World Journal
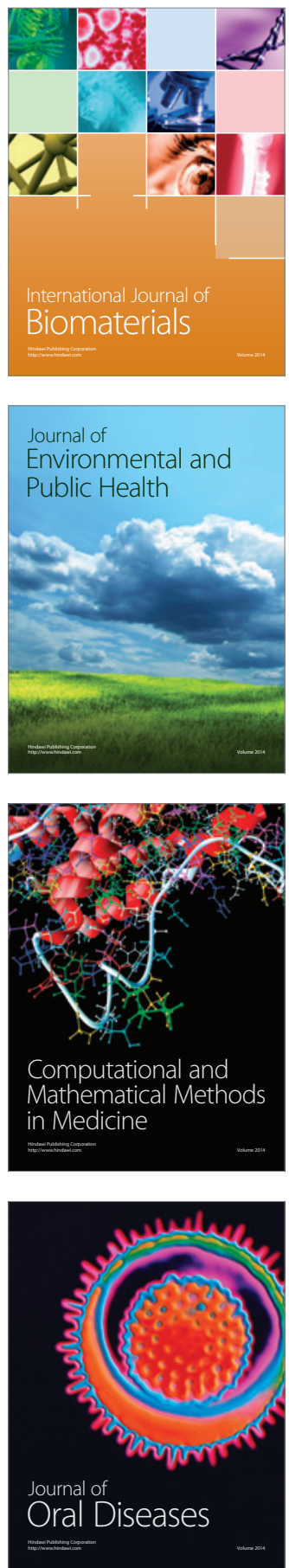
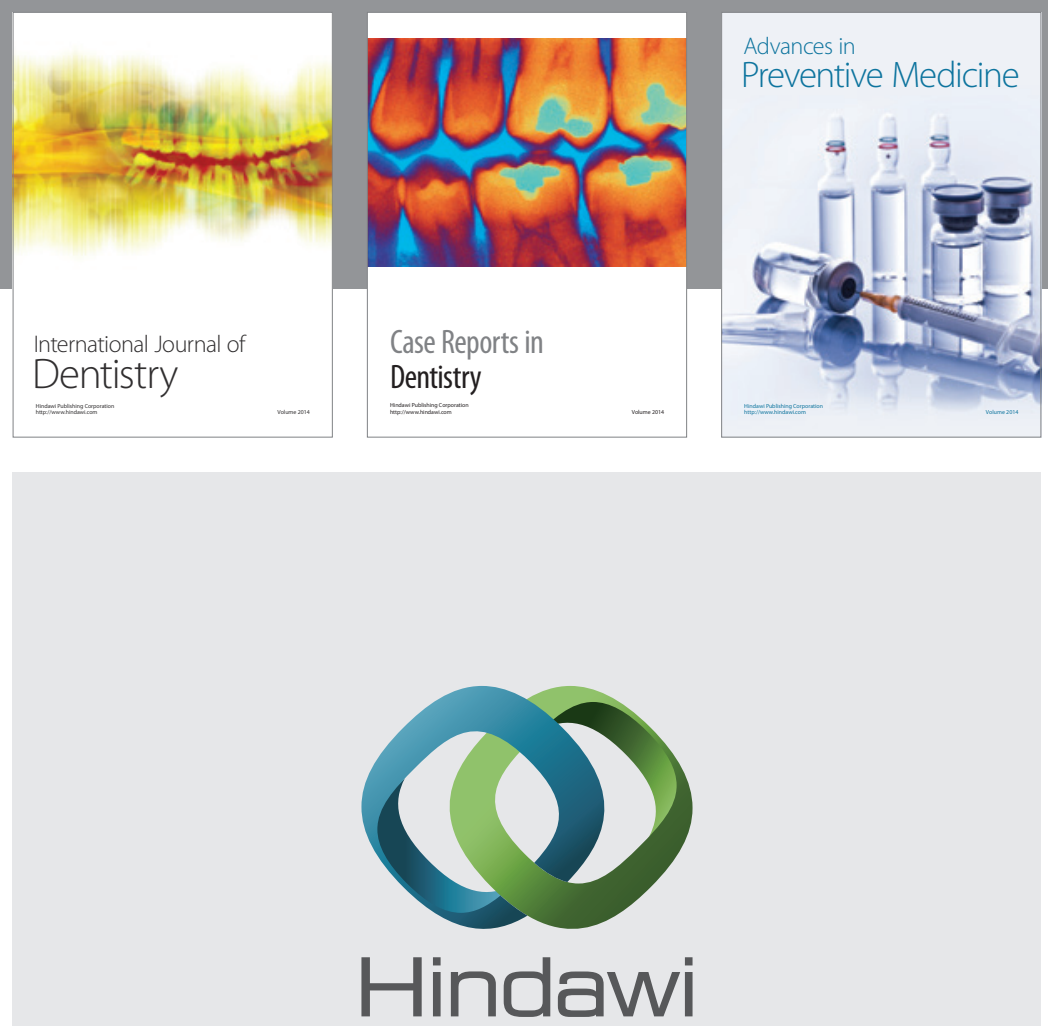

Submit your manuscripts at

http://www.hindawi.com
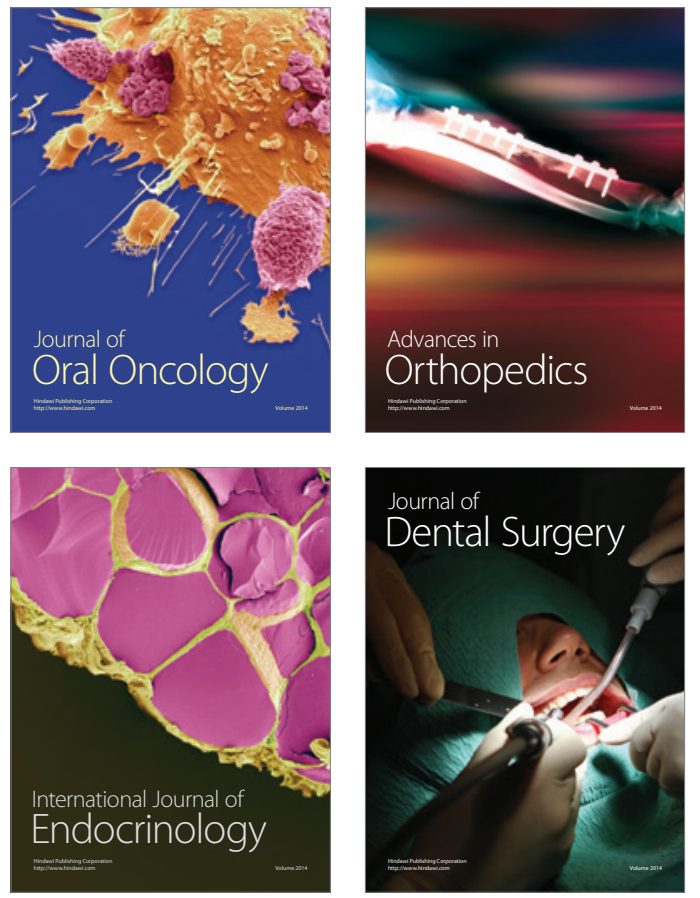
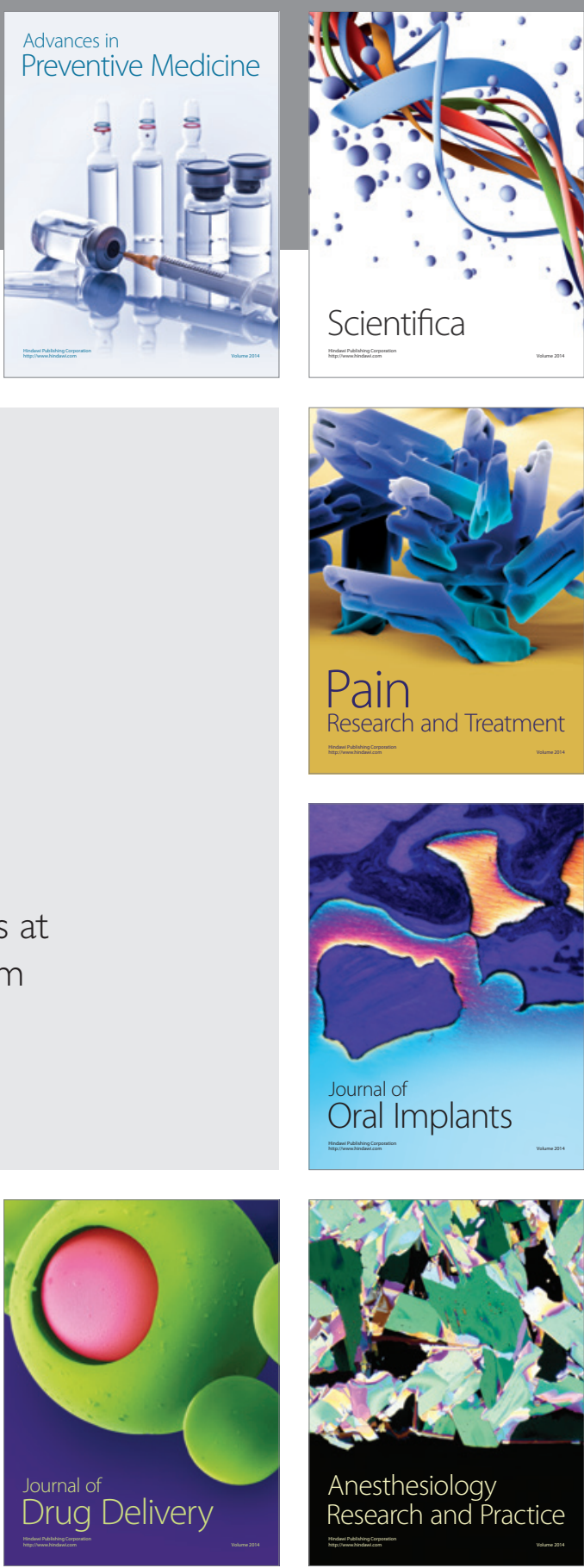

Scientifica
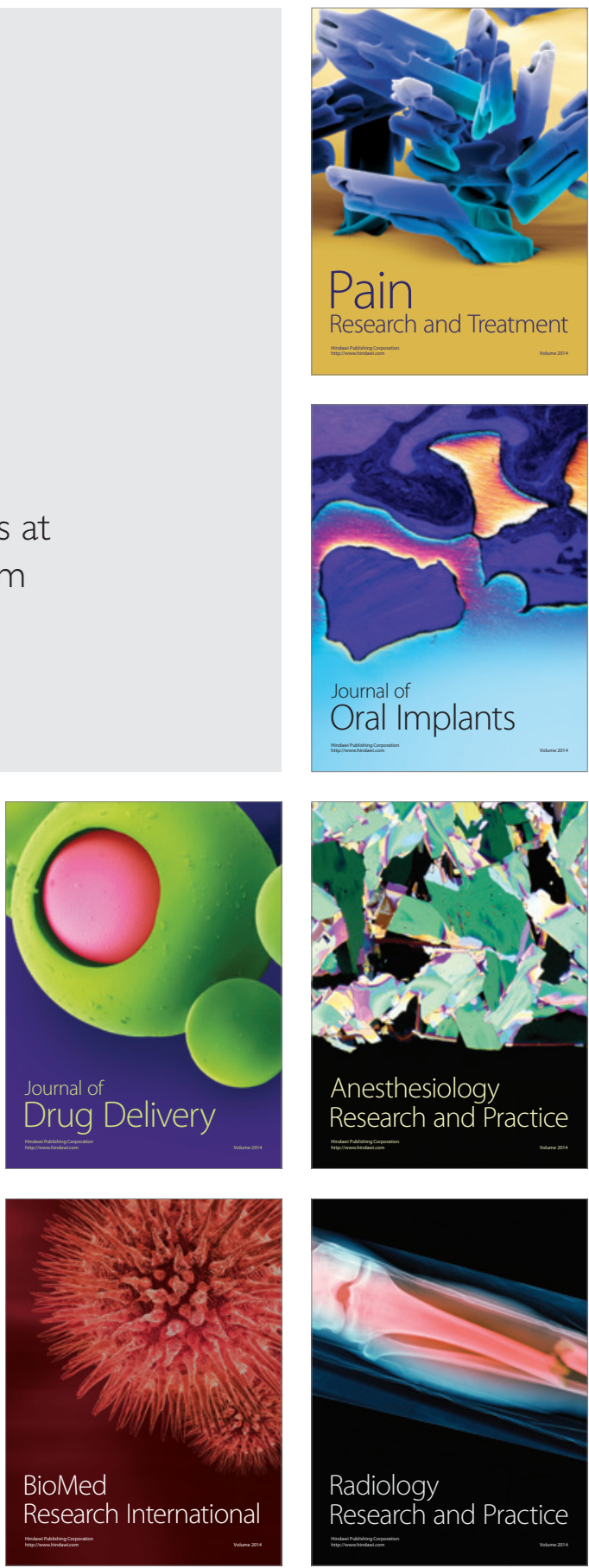